\title{
Osteoarthritis and Articular Cartilage: Biomechanics and Novel Treatment Paradigms
}

\author{
Ray Marks ${ }^{1,2}$ \\ ${ }^{1}$ School of Health \& Behavioral Sciences, York College, City University of New York, New York, USA \\ ${ }^{2}$ Teachers College, Columbia University, New York, USA \\ Email: rm226@columbia.edu
}

Received 14 June 2014; revised 21 July 2014; accepted 25 August 2014

Copyright (C) 2014 by author and Scientific Research Publishing Inc.

This work is licensed under the Creative Commons Attribution International License (CC BY). http://creativecommons.org/licenses/by/4.0/

(c) (i) Open Access

\begin{abstract}
Background: Osteoarthritis is a widespread highly painful disabling age-related disease with no known cure. Although novel strategies for ameliorating osteoarthritic damage abound, it is likely that none will be successful over time if the entire spectrum of the disease and the effects of joint biomechanics on joint tissues are not carefully considered. Objectives: 1) To detail the structure of healthy articular cartilage, the key tissue affected by osteoarthritis. 2) To detail what aspects of cartilage damage best characterize osteoarthritis. 3) To consider the role of biomechanical factors in developing solutions to treat osteoarthritic joint damage. Methods: Literature sources from 1980 onwards that have contributed to our knowledge of the topics relevant to this paper were accessed and retrieved. The data were categorized into four predominant themes and conclusions about the state of our knowledge and future directives were formulated. Conclusions: Osteoarthritis prevalence remains high, and a cure appears elusive. A rich body of data has helped us to better understand the key tissue involved, and suggests a repair process might be feasible, if the basic collective information on the role of biomechanics in mediating or moderating articular cartilage integrity and function is forthcoming.
\end{abstract}

\section{Keywords}

Articular Cartilage, Biomechanics, Osteoarthritis, Prevention, Treatment

\section{Introduction}

Osteoarthritis (OA), a highly prevalent disease, and one that is increasing, is a leading cause of pain, and poor 
life quality throughout the world, affecting several million people [1]. Principally affecting the biomechanical and structural properties of focal regions of the articular cartilage tissue found covering the ends of bones within synovial joints, this debilitating arthritic condition develops slowly over several decades [1] and produces substantial degrees of chronic unrelenting progressive disability in adults over age 65 [2]. In addition to focal damage of the articular tissue which usually progresses to involve the tissue as a whole, OA is usually characterized by detrimental physiological changes of the underlying subjacent bone, as well as the surrounding muscles and soft tissues of the joint [3]. Alone or in combination, all these changes can reduce the ability of the affected joint to absorb joint stresses, and the resultant damage to the joint may consequently severely impair an individuals' ability to carry out his/her normal functions of daily living without compromise, effort and excessive physical stress.

Although little is known about the precise cause of the most common and primary form of OA despite much research, one potential cause of the disease and its associated pathogenic mechanisms, aberrant joint biomechanics may play a considerable role in mediating osteoarthritic joint changes and in preventing treatments designed to improve joint status from having long-term rather than short-term beneficial effects. To improve our understanding of why it is important to acknowledge the role of biomechanical forces in mediating this disease, the present paper attempts to summarize some of the more pertinent advances in our understanding of the complex molecular anatomy and physiology of articular cartilage, the primary tissue affected in OA, and how joint mechanics might have a definite bearing on both causing OA as well as relative failure of attempts to reverse or ameliorate the condition. Finally, the implications of this information for the treatment and prevention of OA are addressed and suggestions are given for some future directions in this important field. Although damage to extraarticular tissues and structures are clearly involved in the osteoarthritic process [3], the present discussion is largely restricted to a discussion of articular cartilage a primary site of osteoarthritic damage. Osteoarthritis is an extremely disabling disease, and affects millions of people worldwide. Since there is no current cure for OA and treatments advocated are not always successful, and are reported in some cases to exacerbate the problem, there is a clear need to continue to examine options for both prevention and intervention that can reduce the burden of the disease. Biomechanical factors are often cited as being important in explaining OA pathology, but are not always incorporated into the solutions advocated for alleviating the prevailing pain and dysfunction.

\subsection{Methods}

Literature sources from 1980 onwards that have contributed to our knowledge of the topics relevant to this paper were accessed and retrieved. The data bases used were pubmed, medline, cinahl, cochrane, academic search premier, and web of science. The key words employed were articular cartilage, biomechanics, and osteoarthritis. Only those papers that focused on the specific topic and the review aims were included. All salient papers were read thoroughly and the relevant information was then either tabulated or incorporated into the document. The data were categorized into four predominant themes and conclusions about the state of our knowledge and future directives were formulated. The focus was on English language papers.

\subsection{Key Objectives}

The key objectives of this review were: to detail the structure of healthy articular cartilage, the key tissue affected by osteoarthritis; to detail what aspects of cartilage damage best characterize osteoarthritis; and to consider the role of biomechanical factors in developing solutions to treat osteoarthritic joint damage.

\section{Articular Cartilage}

\subsection{General Structure}

Under normal physiological conditions, the bony surfaces of all freely moving adult synovial joints are protected by a thin, translucent layer of connective tissue, known as hyaline articular cartilage. Approximately $0.5-7 \mathrm{~mm}$ thick, the key components of articular cartilage are: 1) water which comprises 65 - 80 percent of its wet weight; 2) reinforced collagen which comprises 10 - 30 percent of its net weight; 3) proteoglycan molecules which comprise 5 - 10 percent of its wet weight; 4) cells known as chondrocytes (comprising 1 - 10 percent of the cartilage volume); and 5) small amounts of other noncollagenous proteins, glycoproteins and lipids [4].

In terms of structural organization, chondrocytes, maintain the matrix components under normal, low turnover 
conditions [5], and light and electron microscopic studies of articular cartilage have revealed the existence of a complex layered tissue made up of those constituents mentioned above, in various degrees. As such, the tissue itself can be differentiated broadly into an uncalcified tissue zone and a zone of calcified tissue, which are separated by an irregular zone known as the tidemark. The uncalcified zone is made up of three different types of constituent arrangements as follows: 1) a thin superficial zone constituting approximately two percent of cartilage height that is in contact with the synovial cavity; 2) a deeper transitional zone constituting approximately five percent of cartilage height; 3 ) a third deeper basal or radial zone constituting approximately 92 percent of cartilage height. Beneath this radial zone, and separated from the uncalcified superficial, middle and deep zones by the tidemark is the zone of calcified cartilage [6]. This calcified cartilage zone lies above the subjacent subchondral bone [7] from which it is delineated by a cement line [8] (See Figure 1). All these zones while clearly differentiated are equally important in the ability to protect adjacent bone surfaces as well as the interior of the articular tissue from excessive forces, especially those that might disrupt the viability of the chondrocytes directly, as well the tissue content surrounding these.

\subsection{Cartilage Constituents}

1) Chondrocytes

As revealed by microscopy, cartilage chondrocytes are heterogeneous in shape as well as in function [7]. As the architects of cartilage [9], they are crucial to the viability of the tissue, and whether present as single units, double or multiple linear units, chondrocytes need to be protected from excessive joint forces and/or harmful chemical substrates, in order to function physiologically. In this regard, they are characteristically surrounded by a pericellular or lacunar area whose rich matrix forms a glycocalyx or capsular type structure containing a combination of non-fibrillar collagens such as type IX, non-collagenous proteins such as fibronectin, link protein and laminen, proteoglycans such as hyaluronin and biglycan and a capsule constituted by type II, type VI and type XI collagen fibres [8] [10]. This basket-like network of fine fibrils is termed a chondron [9]. The chondrocytes, in turn are attached to this surrounding matrix and capsule partly through cytoskeletal microfilaments, such as alpha-actinin, talin, and vinculin microfilaments, and partly through cell membrane receptors constituted by glycoproteins such as anchorin II and chondronectin [11] and cell surface adhesion molecules such as CD44 [12]. These receptors are highly sensitive to the mechanical and hydrostatic forces to which they are subject [13] and include those for fibronectin, and type II collagen fragments.

Thus, whether they contain only one isolated chondrocyte or several, the chondrons help to protect the chondrocytes by dampening the forces falling on the tissue during dynamic loading [14]. More specifically, they permit forces falling on the chondrocyte cell membrane, a fluid mosaic composed of large proteins embedded in a thin planar bilayer of aliphatic phospholipid molecules, and comprised by specialized channels and a family of membrane receptors known as integrins [15] to be carefully transmitted and to thereby regulate the flow of nu-

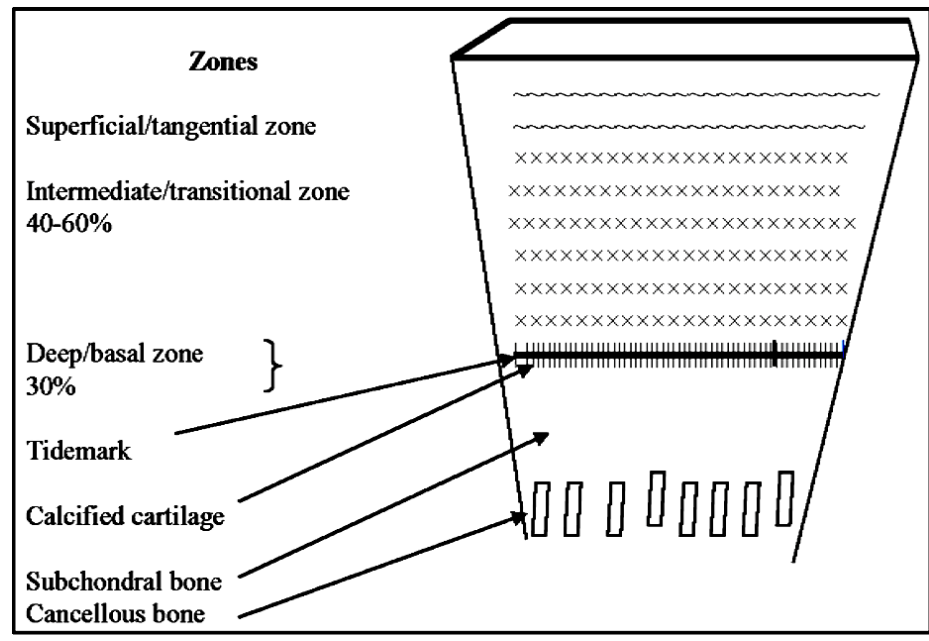

Figure 1. Diagrammatic representation of cartilage structure as outlined by Minus and Nehrer in 1997 [58]. 
trients from the surrounding joint matrix and fluid to the interior of the chondrocyte. Chondrocytes can thus perceive physical signals from their surrounding environment that can have genetic and molecular influences [16]. In addition, by serving as mechanical, electrical and chemical transducers, these membrane channels and receptors, which are selectively sensitive to specific matrical ion and protein (ligands) constituents transport those substrates that mediate profound cellular effects such as proteoglycan synthesis [17]-[19], collagen type II synthesis and cartilage differentiation [15].

While physiologically normal pressures placed on the joint surface can promote cartilage viability [5], unexpected or prolonged alterations from normal pressure can alter chondrocyte gene expression in an unfavourable way [20].

2) Extracellular matrix

The matrical tissue surrounding the cartilage chondrocytes or extracellular matrix, can be differentiated circumferentially into a pericellular region, a territorial matrical area surrounding this and thereafter, by varying expanses of interterritorial matrix [8]. This matrix framework, which protects the chondrocytes from the harmful effects of excessive loading impactsis composed largely of water, and a proteoglycan called aggrecan that forms the bulk of the articular tissue, along with a variety of collagens, glycoproteins and noncollagenous proteins.

The hydrous or water component of articular cartilage which forms its major constituent, contains gases, small proteins, metabolites, and a high concentration of positive ions or cations. While much of this water is bound to the proteoglycan and collagen molecules mentioned above, at least some of this water can move freely in and out of the tissue [19]. The resultant alterations in tissue ionic content and degree of resulting osmolarity that occurs due to changes in ionic concentration as a result of the movement of water in response to loading and unloading of the joint are of high import in the maintenance of the tissues' load bearing properties [21].

The two classes of proteoglycans that constitute the bulk of articular cartilage, the aggrecans and small nonaggregating proteoglycans, including biglycan, fibromodulin, and decorin [21] contain extensive negatively charged side-chains constituted by two glycosaminoglycans, chondroiten sulphate and keratin sulphate. These disaccaride constituents of aggrecan are bound co-valently to a protein core by link protein and to strands of hyaluronic acid, another proteoglycan to form stable matrical aggregates [22] (See Figure 2). These disaccaride units, which are associated with negatively charged carboxyl or sulphate groups and whose side chains repel each other, tend to attract cations including water molecules. This ability to bind water plays a fundamental role in facilitating load transmission, and chondrocyte function [5].

In addition to aggrecan, vital to the structural integrity of articular cartilage is type II collagen, which exhibits a highly differential degree of ordering within the articular tissue consisting of: 1) fine collagen fibrils located at the articular tissues surface which are arranged tangentially, thick collagen bundles lying subjacent and parallel to its surface, 2) coarse radially oriented bundles forming a meshwork in the intermediate or transitional zone of

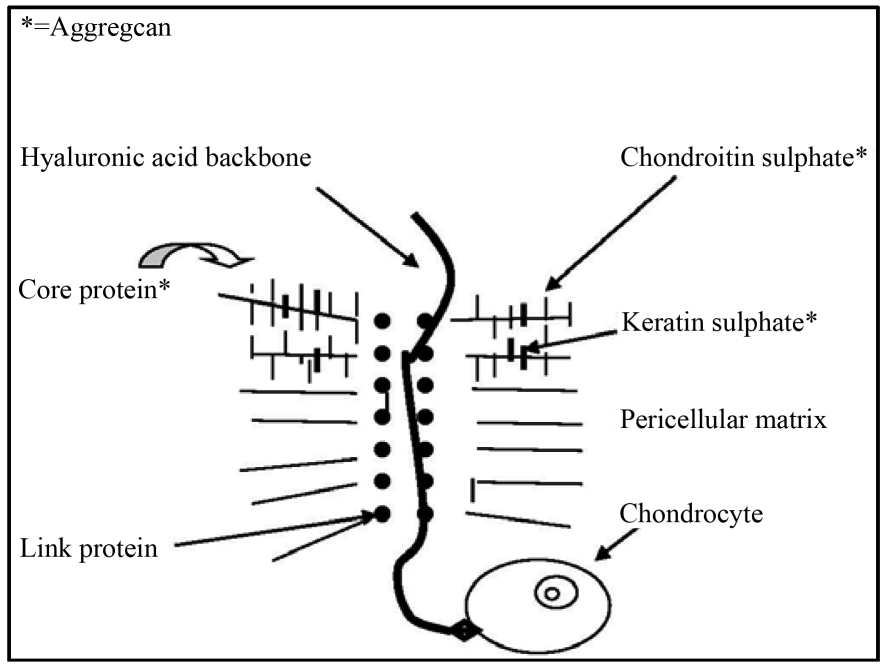

Figure 2. Graphic representation of structure of proteoglycan molecules of articular cartilage as adapted by the author from Aigner et al. [59]. 
articular cartilage, 3) random or coiled radial fibres in the deep zone which link the uncalcified cartilage to the underlying calcified cartilage, and 4) vertical fibres in the calcified cartilage which link the cartilage to the underlying subchondral bone [19].

The integrity of this collagen mesh, differentially ordered to conform with the concentric cartilage subdivisions observed in the horizontal plane [8], is maintained by interlinking molecules such as type IX collagen, and/or by viscous forces between the fibrils and surrounding ground substance [19]. Although highly resistant to enzymatic cleavage with an extremely slow metabolism, cartilage collagen is readily digested by enzymes such as metalloproteases and cathepsins. On excessive exposure to such degradative enzymes, numbers of type II collagen fibres may be reduced, as may cartilage integrity, and the normal production of Type IX, XI, XII, V and VI cartilage collagens may be diminished.

In general however, collagen interacts closely with the proteoglycans of the matrix and this restraining meshwork, contributes to the unique loading properties of cartilage tissue [8] [21] and may help to restrict collagen fibrillogenesis, and influence chondrocyte cell function [8] [21], and the forces acting on cartilage must fall within a range suited to the continued vitality and viability of the cartilage cells [23], as well as the collagen framework. That is, they must be of sufficient amplitude to provide for a nutritional supply from synovial fluid to the avascular chondrocyte matrix, but must not be excessive so as to produce cellular or matrix necrosis. Where mechanical stress on the cartilage falls outside the optimum range, degenerative processes may be expected to ensue, regardless of age, and growth hormone availability. This may involve mechanical damage to the collagen meshwork, proteoglycan dysfunction, chondrocyte metabolic alterations, and/or synovial degradative enzyme production.

\section{Osteoarthritic Cartilage}

Various studies have attempted to examine processes that lead to articular cartilage destruction in both human and animal model contexts. In animal models as well as in human OA, researchers have observed that increased hydration of the cartilage matrix, an early and key event, may follow disruption of type II, type IV and/or type IX collagen fibres as a result of aging and fatigue failure, synovium and/or chondrocyte mediated enzymatic degradation of collagen [24] [25]. As well, debonding of the collagen-proteglycan interface leading to swelling of the cartilage matrix could reduce the stiffness of the tissue without any collagen network disruption [26]. Overall, the disruption of collagen fibres and/or collagen crosslinks from any cause is likely to deplete the elasticity of cartilage, and reduce its resistance to weightbearing [27], while facilitating fragmentation of the cartilage proteoglycans which causes secondary inflammatory and degradative sequelae.

As well as the disruption of type II collagen, and type IX collagen [24], traces of collagen Type I, variants of collagen Type II [7] and Type X collagen and Type I and III collagens, proteoglycans with an atypical glycosaminoglyan content may contribute to osteoarthritic joint disease [28]. In this respect, Type $\mathrm{X}$ collagen which is not normally found in healthy uncalcified cartilage, and is unable to form a fibrillar meshwork comparable to that of Type II/IX/XI collagen, may weaken the loading capacity of the articular cartilage matrix, perhaps preparing it for, or contributing to, it's excessive hydration and/or calcification.

Also damaged may be fine collagen filaments containing Types VI and IX collagen located at the pericellular level, together with proteoglycans and collagen that protect the chondrocyte from stress. The pericellular fibrils while normally deficient towards the surface of the articular tissue forming pericellular channels between the general matrix and the lacunae surrounding the chondrocyte [29] may thus lose their ability to afford protection of cytoplasmic organelles from extraneous mechanical forces. Alterations in transduction processes across the cell membrane by virtue of these changes in pericellular collagen, as well as direct damage to the glycoproteins, anchorin and chondronectin and the integrins, which are possible mechanotransducers in addition to promoting cell adhesion may further disrupt cartilage contiguity.

In addition to disruption of the predominant proteoglycan mediating the absorption of joint stresses, namely aggrecan, the synthesis of two smaller proteoglycan units found in articular cartilage, namely decorin located on the surface of the collagen fibers and biglycan which may be involved in the inhibition of cell adhesion and migration [11] may be impaired with deleterious consequences. Accompanying these changes may be excessive degradative enzyme production, which further damages collagenous and non-collagenous matrix constituents and promotes catabolic activation [9]. The articular cartilage matrix normally partly constituted by collagen arcades and meshwork which controls loading responses and the diffusion of metabolic solutes into and from the cartilage tissue may hence be markedly disrupted, and once disrupted may not return to its original state [9]. 
Other research shows that any disruption of the adhesive associations between the chondrocyte and its pericellular matrix for example by dissociation of fibronectin may produce a phenotypic transformation of the chondrocyte cell and an alteration in its metabolic state [30], with higher rates of production of proteoglycan, hyaluronate and specifically the fibronectins [31] and COMP non-collageous proteins [32]. This hypermetabolic state may represent an attempt by the chondrocyte to mediate cartilage repair.

However, due to their great affinity for water, the increased deposition of proteoglycan molecules that can ensue could cause further cartilage loading impairments [33]. As well, increases in the rate of proteoglycan synthesis with length modifications which increases their hydrodynamic size plus synthesis of chondroiten sulphate chains which differ structurally from normal chains have been observed [34] and this altered conformation of the hydrophilic proteoglycans may permit their further hydration [35].

In addition to the cartilage thus formed being less suitable for its physiological role, fibronectin a matrix molecule which has a broad range of cellular functions including repair and assembly of cartilage oligomeric high- $\mathrm{M}_{\mathrm{r}}$ matrix protein (COMP) and thromboplastin [4], may be disassociated. At the same time, the three long-chain proteoglycan molecules, namely, chondroitin-4-sulphate, chondroitin 6-sulphate and keratin sulphate normally assymetrically distributed and covalently bound to a protein core and long filaments of hyaluronic acid may be replaced by disaggregated proteoglycans of immature quality despite their increasing production. This may be due to structural defects in link protein and/or the G1 or globular domain of the protein core of aggrecan which interacts noncovalently with hyaluronic acid [11]. The hyaluronic acid backbone of osteoarthritic cartilage, may also be structurally modified despite its increasing production. The disaggregated matrical proteoglycan complexes, no longer able to effectively constrain water within the fragmented and fibrillated collagen meshwork, may not protect the matrix against loading stresses, at all adequately [36]. The abnormal proteoglycan fragments may also fail to interact optimally with intact collagen fibres thus reducing the ability of the tissue to protect the joint from excessive shear and compressive joint stresses [37].

As well as the abovementioned deleterious processes, biomechanical damage to the phospholipid membrane, the destruction of surface receptors for hyaluronan and interleukin antagonist receptors, plus the release of prostaglandins which stimulates protease activity may initiate a further degradative cascade along with damage to intracellular chondrocyte component [5] [38]. A similar cascade could occur due to biomechanically induced microfractures in the subchondral bone in response to aberrant joint loading which vascularises the cartilage and reduces the efficacy of protease (tissue inhibitor metalloproteinases TIMP) and endothelial-cell growth factor inhibitors [39].

\section{Possible Pathogenic Pathways Involved in Osteoarthritis}

While the most likely event producing osteoarthritic damage is unclear, altered cell-matrix interactions and/or disruption of the extracellular matrix molecules, and the consequent failure of newly formed proteoglycans and hyaluronate to aggregate seem to play a major role. While damage may be caused by trauma, or overloading of normal or abnormal cartilage, the presence of persistent abnormal joint mechanics that can result regardless of the actual cause of the problem, is likely to interact adversely with the damaged tissue in terms of preventing aggregation-or causing defects in link protein molecules and the binding of hyaluronate to aggrecan molecules.

The precise structure of the newly formed aggrecan and hyaluronate molecules may also be abnormal due to stress-induced alterations in chondrocyte genetics, precluding their effective aggregation. Thus the cartilage so formed may not effectively absorb high contact stresses consequent to mechanical loading, particularly if this is excessive in magnitude and/or duration, or suboptimal, thus negatively impacting adequate chondrocyte nutrition, as well on the intact extracellular proteoglycan-hyaluronic acid and collagen complexes and their chondrocyte adhesions.

Moreover, as with chondrocytes exposed to cell cultures devoid of extracellular matrix, the chondrocytes in these areas of matrical damage may undergo mitotic changes and/or migrate to form clones [40]. That is, they may proceed to carry out an attempted reparative process as suggested by Brocklehurst et al. [41], but although increased in number, may actually dedifferentiate. With their synthetic capability modified, immature, rather than mature, cartilage contents may develop. Altered as well, may be the balance of enzymes which normally inhibit catabolism-especially collagen cleavage, such as the Tissue Inhibitors of Metalloproteases (TIMPS) and its antagonistic Interleuken-1 receptors (Il-1 RA), and possibly some component of the Transforming Growth Factor-B (TGF-B) cell membrane receptors. Upregulated however, may be the production of chondrocyte and 
synovial cell tissue metalloproteases, elastases, stromelysin enzymes, aggrecanases, and collagenases, stromal cell derived factor-1 (SDF-1) [42], possibly in an attempt to catabolize the altered collagen being produced, or because synovial membrane inhibitors (e.g. catabolin) are depleted.

Alternatively, neovascularisation from the underlying bone [24] may activate previously inactive cytokines [24] as might destructive alterations in Interleukin (IL)-6 and its soluble cell membrane receptor [43] causing pain, swelling, and stiffness [13]. Roughley et al. [44] reported that osteoarthritis appears to be associated withproteolytic degradation of its constituent proteoglycan aggregates, and that degradative changes in link protein have been characterized. Although Sokoloff argued that it is difficult to identify abnormalities confined to the cartilage as being solely responsible for OA [45], direct trauma affecting the chondrocyte cell membrane, may increase prostaglandin E activity and protease synthesis [38], and if pressure on the chondrocyte is not normalized, research by Benya and Shaffer [46] suggests the normal phenotype may be lost and replaced by a complex collagen phenotype consisting predominately of type I collagen and a low level of proteoglycan synthesis. According to a review by Mobasheri et al. [47] these types of cartilage alterations can foster synovial inflammation, creating a vicious circle of joint degradation. Ultimately, the fragmentation of the proteoglycans and their resultant inferior structure may stem from direct damage to intracellular structures such as microtubules and the golgi apparatus. A synovial inflammatory response which compounds this situation would be expected to produce a further cascade of destruction and ultimate death of viable chondrocytes due to release of nitric oxide, hydroxyl and superoxide radicals and with this more widespread cartilage damage and erosion. This is supported by findings of Petterson et al. [48] that human chondrocytes express receptors mediating apoptosis and can be subject to cell death after exposure to tumor necrosis factor alpha. These researchers further suggested that these death receptors and their respective ligands may play a crucial rule in both cartilage generation as well as destruction.

However, since most afflicted individuals will at this stage be experiencing pain and receiving medications to control their pain and inflammation, the likelihood exists that any primary causative factor underlying the condition, such as abnormal prolonged high intensity joint loading that can increase the expression of inflammatory mediators [5], will simply be overlooked and further aberrant loading will ensue, thus perpetuating the problem as outlined in Figure 3 as indicated by genetic models that show altered biomechanics alone can produce spontaneous or accelerated OA [13]. Untreated, this situation is hence likely to contribute to the diseases' progress-

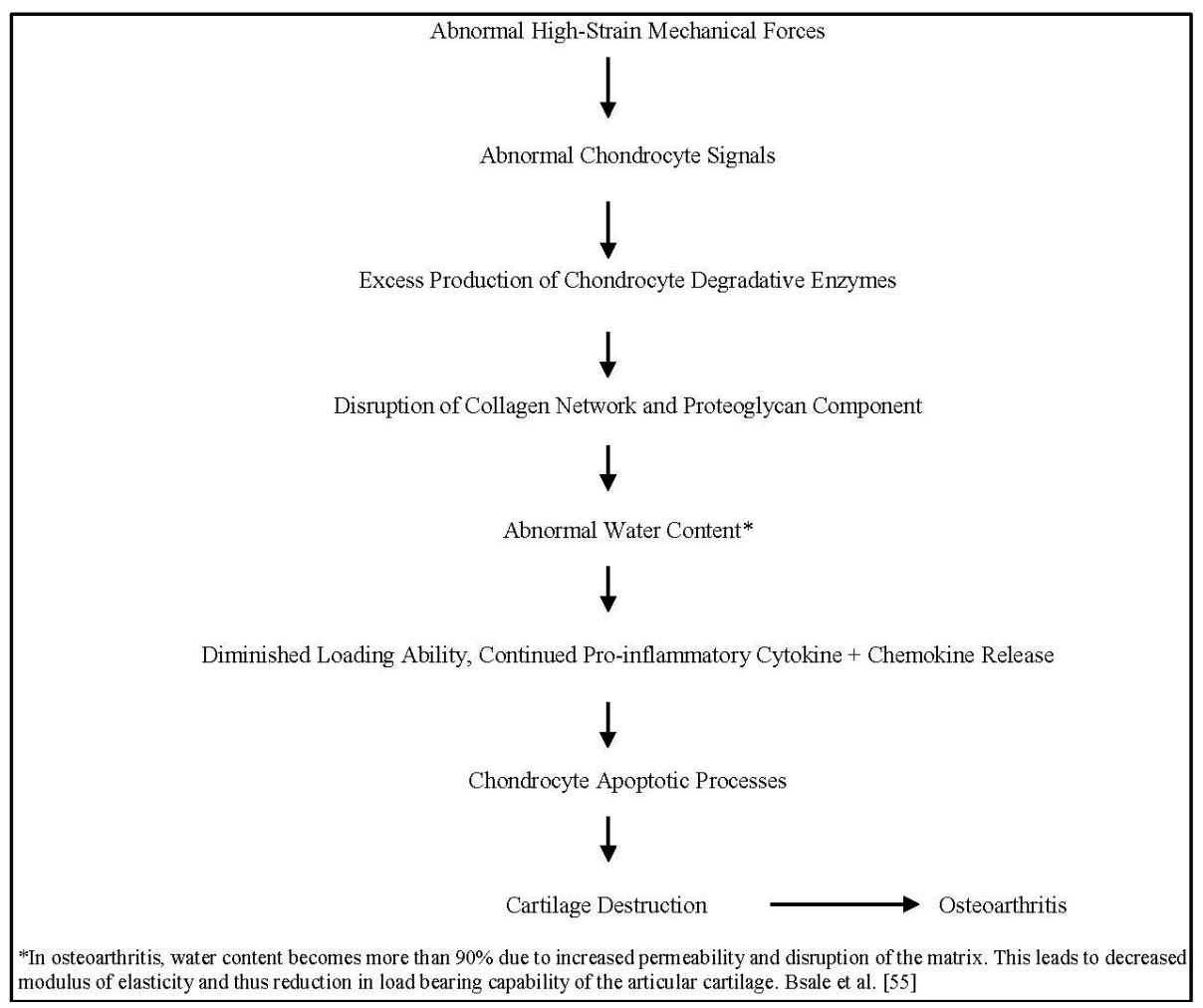

Figure 3. Hypothetical pathway of osteoarthritis destruction from a biomechanical standpoint. 
sion and may hasten the onset of the late or end stages of the disease where cartilage may be totally destroyed, especially in cases of obesity, where excess mechanical load and/or adipokine release can serve to raise the level of destruction in both weight bearing and non weightbearing joints [3].

\section{Treatment to Minimize Osteoarthritic Destruction}

In light of the complexity of the osteoarthritic process, treatments to minimize osteoarthritic cartilage destruction must be carefully conceived. In particular, because the disease process in OA is greatly influenced by the prevailing status of the neuromuscular system and its ability to protect the joint and underlying subchondral bone, the role of biomechanics in the disease process cannot be ignored. Moreover, without careful consideration of how joint biomechanics influences articular cartilage viability, the likelihood is that focal treatments of the articular tissue, carried out in isolation will fail to completely ameliorate the disease, and may not prevent its reoccurrence at the same joint or distant joints with time. For example, given the physiological dependence of normal chondrocyte physiology on extrinsic factors such as joint motion and joint loading which facilitates cartilage nutrition, transplantation of cartilagenous tissue, resurfacing of affected joints or restoration of articular surfaces using growth factors and other methods of stimulating cartilage is bound to have no durable effect, if joint deformities and capsular contractures persist, muscle strength and coordination is suboptimal, and joint laxity remains excessive or and untreated. The same idea would apply to the application of cartilage implants, or targeted cell-mediated gene transfer, which would ideally combine a supply of chondroprogenitor cells with the production of therapeutic factors directly at the lesion site [49]. Moreover, to be optimally effective in the case of implants, the structural heterogeneity of articular tissue at the cellular level would not only have to be precisely reproduced in three-dimensions, but also very carefully mapped to the configuration of any defective region(s).

In addition, even with some form of enzymatic control, in the face of continued cartilage fragmentation, and or cell membrane damage it is difficult to see how all immunogenic or related apoptotic responses and those involving production of reactive oxygen and nitric acid radicals that can impair articular chondrocyte functions can be obviated. It is also not clear from the pathogenesis of the disease, what proteolytic enzymes might be targeted to reduce matrix catabolism, and for what durations? Likely candidates, the metalloproteinases, the cytokine-interleukin-1, tumor necrosis factor alpha, lysosomes, the Cathepsins may need to be targeted differentially or contemporaneously depending on disease stage and sites of destruction. Alternately, Roughly, Nguyen and Mort [44] found cartilage remaining in osteoarthritic joints did not show more extensive proteolysis than its normal counterpart. Evidence also shows that reducing inflammatory cytokine levels effects osteoarthritis symptoms, but not the disease progression [42].

It is thus open to speculation whether antiiflammatory interventions or enzymatic blockers can prevent cartilage destruction in the long term, particularly upon cessation of their usage or in absence of any ongoing anabolic reparative processes or any respite from abnormal loading. Indeed, as indicated by Sokoloff [45], OA may not be so much the inability of the articular surface to repair itself, but an aberration of the repair process resulting from persistent abnormal loading.

Thus unless multiple therapeutic strategies including periods of rest so that damaged cartilage can rehydrate and mount a reparative process, from the earliest point in time, along with other biomechanical strategies, and these are not applied insightfully and consistently, possibly along with growth factors to enhance cell function, treatment will likely prove less than optimal. That is, if all sources of abnormal mechanical loading are not minimized and rendered as close to optimal as possible, all extra articular interventions, particularly those that may have the capacity to modulate chondrocyte synthetic mechanisms and biological resurfacing of damaged cartilage by new bone growth or implants are likely to fail. In addition, although Stockwell [29] has opined that OA has no systemic effects, the lack of mobility and dysfunction that usually accompanies untreated or inappropriately treated osteoarthritic joint disease can surely affect cardiovascular health as well as bone health detrimentally and therefore if unattended to may result in further damage, joint dysfunction, and immobility due to pain even if cell-based repair strategies are initially favorable. The use of various remedies applied in isolation to quell pain have largely failed as well [see review in citation 1], possibly because in the absence of pain, and no biomechanical intervention, more forceful or prolonged loading of the damaged joint causes more harm than good.

In that this could be an added complication in so far as producing suboptimal plasma and synovial fluid os- 
molarity states that favor chondrocyte physiology, this potentially detrimental situation may be effectively obviated by interventions which reduce pain and joint loading but which encourage moderate forms of exercise and maintenance of joint mobility and sensibility. Since chondrocytes are also found to recover their normal protein synthesis when osmolarity of the surrounding fluid is reduced from any dangerously high osmolarity levels [40], interventions which reduce joint compressive forces, especially those which create focal joint stresses may prove especially helpful. Interventions which promote cell shape in its rounded form are also likely to promote maintenance of the phenotype [46] and possibly explain why efforts to apply distraction may prove highly beneficial to the osteoarthritic damaged joint.

Finally, to reduce the overall burden of osteoarthritis, and enable biological and surgical as well as gene therapies to be efficacious, where desirable, multiple preventive strategies highlighted in Figure 4, are likely to prove beneficial for purposes of primary, secondary, as well as tertiary prevention, and should not be underestimated in efforts to foster optimal joint function, across the lifespan, regardless of level of joint pathology.

\section{Conclusions}

Articular cartilage, covering the needs of freely moving joints is a highly active tissue and one that depends largely on mechanical stimuli for its growth and maintenance [47]. Aberrant loading, on the contrary, can lead to cartilage damage as supported by numerous loading studies ranging from immobilization to excessive weightbearing.

Although less is known about the molecular mechanisms underlying these mechanical effects, recent studies from molecular biology and cell membrane signalling pathways suggest mechanical stimuli and others can activate cartilage cellular mechanisms differentially.

In this regard, Qwing et al. [50] suggest that several factors are involved in the osteoarthritic disease process, including the degradation of proteoglycans, abnormal loading effects that may trigger an imbalance of chondrocyte anabolic and catabolic activities, and structural damage of the proteoglycans and collagen fibrils that triggers an immunological reaction and consequently the accelerated degradation of proteoglycans.

In terms of biomechanics, research shows cartilage tissue can remodel its extracellular matrix in response to alterations in functional demand, and that changes in the properties of the pericellulur matrix with osteoarthritis may alter the stress-strain and fluid-flow environment of chondrocytes as well as its loading features. Moreover, excessive compression that overloads the integrity of the cartilage matrix may in turn, and causes cell membrane damage and eventually cell death after which tumor necrosis factor and interleuken-1 foster stress-injury-related proteins release [51].

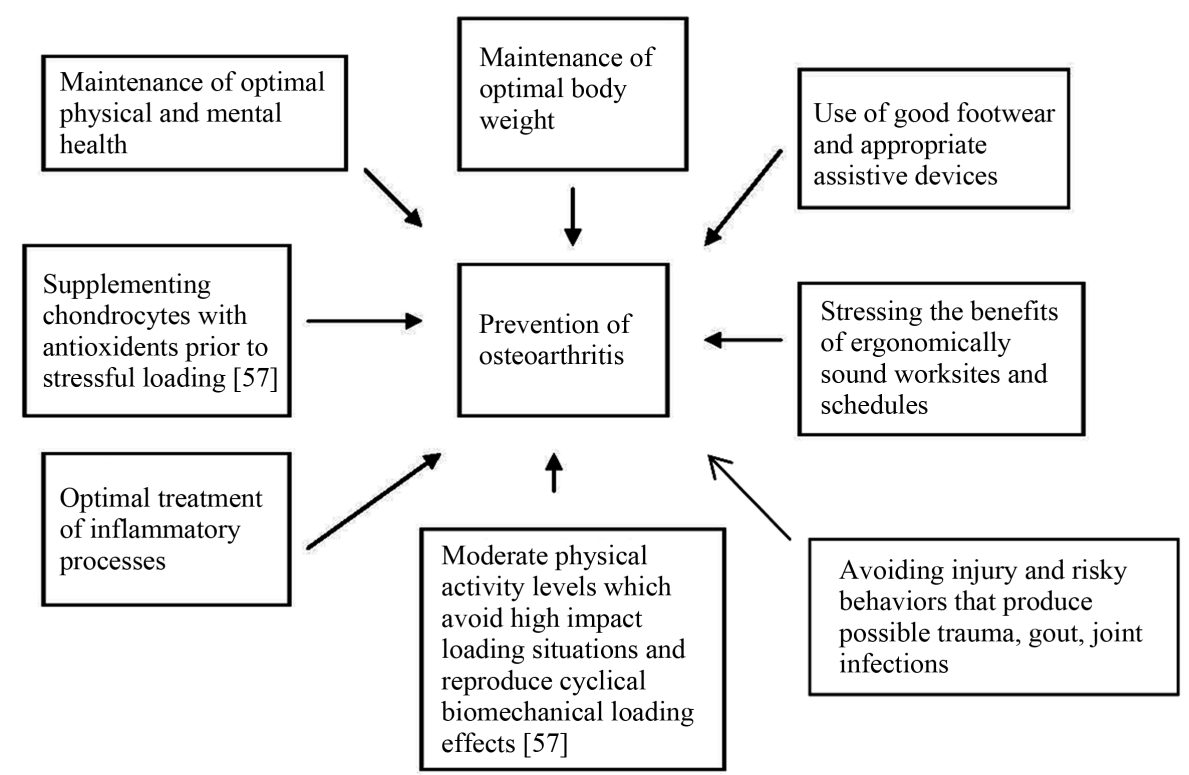

Figure 4. Suggested strategies to prevent abnormal joint loading and excess destruction in people with osteoarthritis. 
Given the poor capacity of articular cartilage for repair, and that millions of older adults suffer immense pain as a result of OA, and that treatments commonly directed to reduce pain may not focus on identifying the cause of the problem, a better understanding of the pathways that foster normal cartilage physiology as well as those that are potentially involved in mediating cartilage pathology is indicated. As pointed out by Aigner et al. [52] very often the primary targets of osteoarthritis therapy, the extracellular matrix and the cartilage chondrocytes, do not take into account other joint tissues that are also important for fostering the disease [45], or the movement patterns that lead to the disease or both and these elements are located outside the articular tissue. Although implantation of mesenchymal stem cells [53] or gene therapy [54] or implantation therapy [55] have been advocated, recent research further reveals that osteoarthritic chondrocytes may undergo genetic alterations that heighten the release of degrading enzymes [56], and reactive oxygen species [57].

Moreover, autogenous osteochondral arthroscopically implanted grafts, or open implantation of lateral patellar facet, requires violation of subchondral bone, risk of viral transmission of disease and low chondrocyte viability, in addition to removal of host bone for implantation [58], even though bone integrity is vital to joint function, and is often believed to be the precursor of OA, rather than the outcome of this disorder.

In addition, cellular implants may not be helpful if the heterogeneity of the chondrocytes is not accounted for, and severe structural changes in the extracellular matrix are not addressed. The chondrocytes may also display abnormalities during osteoarthritic cartilage degeneration, and may continue to be exposed to additional stimuli such as nonphysiologic loading conditions and byproducts of matrix destruction, as well as abnormal levels of cytokines and growth factors [59]. This exposure can lead to a structured cellular response pattern that may be highly detrimental to the cartilage tissue if not accounted for. Consequently, even if it appears cartilage tissue might serve as the key target for ameliorating this condition, the role of joint biomechanics and biomechanical transduction pathways and their relationship to OA processes cannot be ignored if treatments such as the implantation of mesenchymal stem cells [53] or gene therapy [54] are to be helpful. That is surgical implantation designed to foster physiologic repair using mesenchymal stem cells or chondrocytes delivered surgically in an ex vivo-derived matrix, in vitro manipulation of cells with growth factors, would still be subject to the effects of intrinsic and extrinsic mechanical stimuli [58]. By envisioning how joint biomechanics can influence molecular events at the chondrocyte cell membrane level as well as at potential gene expression levels, which in turn influences cartilage composition and joint anatomy and physiology, researchers and clinicians will undoubtedly be in a better position to advance the state of the art of preventing and treating an osteoarthritic joint, as outlined by Brady et al. [60], notwithstanding the importance of considering the bone, and synovial tissues among others, and are encouraged to do so. All aging populations worldwide, who are at an especially high risk for this condition, given the prevalence of obesity, and sedentary lifestyles among adults today, as well as policy makers who must fund their needs, could be ably served in this regard.

\section{References}

[1] Martel-Pelletier, J., Wildi, L.M. and Pelletier, J-P. (2012) Future Therapeutics for Osteoarthritis. Bone, 51, $297-311$. http://dx.doi.org/10.1016/j.bone.2011.10.008

[2] Breedveld, F.C. (2004) Osteoarthritis-The Impact of a Serious Disease. Rheumatology, 43, i4-i8.

[3] Lajeunesse, D., Pelletier, J-P. and Martel-Pelletir, J. (2005) Osteoarthritis: A Metabolic Disease Induced by Local Abnormal Leptin Activity? Current Rheumatology Reports, 7, 79-81. http://dx.doi.org/10.1007/s11926-005-0057-0

[4] Kuettner, K.E., Aydeotte, M. and Thonar, E.J.-M.A. (1991) Articular Cartilage Matrix and Structure: A Mini Review. Journal of Rheumatology, S27, 46-48.

[5] Goldring, M.B. and Otero, M. (2011) Inflammation in Osteoarthritis. Current Opinion in Rheumatology, 23, 471-478. http://dx.doi.org/10.1097/BOR.0b013e328349c2b1

[6] Passiu, G. and Carcassi, U. (1989) Recent Trends in the Pathophysiology of Osteoarthritis. Rays, 14, 351-360.

[7] Hamerman, D. (1989) The Biology of Osteoarthritis. New England Journal of Medicine, 320, 1322-1330. http://dx.doi.org/10.1056/NEJM198905183202006

[8] Poole, C.A. (1987) Articular Cartilage Chondrons: Form Function and Failure. Journal of Anatomy, 191, 1-13. http://dx.doi.org/10.1046/j.1469-7580.1997.19110001.x

[9] Muir, H. (1995) The Chondrocyte, Architect of Cartilage. Biomechanics, Structure, Function and Molecular Biology of Cartilage Matrix Macromolecules. Bioessays, 17, 1039-1048. http://dx.doi.org/10.1002/bies.950171208

[10] Gardner, D.L. (1994) Problems and Paradigms in Joint Pathology. Journalof Anatomy, 184, 465-476. 
[11] Kuettner, K.E. (1992) Biochemistry of Articular Cartilage in Health and Disease. Clinical Biochemistry, 25, $155-163$. http://dx.doi.org/10.1016/0009-9120(92)90224-G

[12] Ishida, O., Tanaka, Y., Morimoto, I., Takigawa, M. and Eto, S. (1997) Chondrocytes are Regulated by Cellular Adhesion through CD44 and Hyaluronic Acid Pathways. Journal of Bone and Mineral Research, 12, 1657-1663. http://dx.doi.org/10.1359/jbmr.1997.12.10.1657

[13] Goldring, M.B. (2012) Articular Cartilage Degradation. Hospital for Special Surgery Journal, 9, 7-9.

[14] Poole, C.A., Flint, M.H. and Beaumont, B.W. (1987) Chondrons in Cartilage: Ultrastructural Analysis of the Pericellular Microenvironment in Adult Human Articular Cartilages. Journal of Orthopedic Research, 5, 509-522. http://dx.doi.org/10.1002/jor.1100050406

[15] Salter, D.M., Godolphin, J.L. and Gourlay, M.S. (1995) Chondrocyte Heterogeneity: Immunohistoloically Defined Variation of Integrin Expression at Different Sites in Human Fetal Knees. Journal of Histochemistry and Cytochemistry, 43, 447-457.

[16] Guilak, F. (2011) Biomechanical Factors in Osteoarthritis. Best Practices in Research and Clinical Rheumatology, 25, 815-823. http://dx.doi.org/10.1016/j.berh.2011.11.013

[17] Knudson, C.B. (1993) Hyaluronan Receptor Directed Assembly of Chondrocyte Pericellular Matrix. Journal of Biological Chemistry, 120, 825-834.

[18] Lapadula, G., Iannone, F., Zuccaro, C., Grattagliano, V., Covelli, M., Patella, V., et al. (1997) Integrin Expression on Chondrocytes: Correlations with the Degree of Cartilage Damage in Human Osteoarthritis. Clinical and Experimental Rheumatology, 15, 247-254.

[19] Stockwell, R.A. (1974) Cellular Aspects: The Chondrocyte Population and the Chondrocyte. In: Ali, S.Y., Elves, M.W. and Leaback, D.H., Eds., Normal and Osteoarthritic Articular Cartilage, Institute of Orthopaedics, London, 461-486.

[20] Sironen, R.K., Karjalainen, H.M., Elo, M.A., Kaarniranta, K., Törrönen, K., Takigawa, M., et al. (2002) cDNA Array Reveals Mechanosensitive Genes in Chondrocytic Cells under Hydrostatic Pressure. Biochimica et Biophysica Acta (BBA)—Molecular Cell Research, 1591, 45-54. http://dx.doi.org/10.1016/S0167-4889(02)00247-1

[21] Buckwalter, J.A. and Mankin, H.J. (1998) Articular Cartilage: Tissue Design and Chondrocyte-Matrix Interactions. AAOS Instructional Course Lectures, 47, 477-486.

[22] Muir, I.H.M. (1980) The Chemistry of the Ground Substance of Joint Cartilage. In: Sokoloff, L. Ed., The Joints and Synovial Fluid, Academic Press, New York, 27-94. http://dx.doi.org/10.1016/B978-0-12-655102-0.50008-4

[23] Unsworth, A. (1984) Some Biomechanical Factors in Osteoarthritis. Journal of Rheumatology, 23, 173-176. http://dx.doi.org/10.1093/rheumatology/23.3.173

[24] Choi, W. (1996) Alterations in Articular Cartilage of the Rabbit Mandibular Condyle Following Surgical Induction of Anterior Disc Displacement: Light and Electron Microscopic Immunocytochemistry Using Colloidal Gold Conjugates. Ph.D. Thesis, Medical College of Georgia, Augusta.

[25] Eyre, D.R. (1991) The Collagens of Articular Cartilage. Seminars in Arthritis and Rheumatism, 3, 2-11. http://dx.doi.org/10.1016/0049-0172(91)90035-X

[26] Schwartz, M.H., Leo, P.H. and Lewis, J.L. (1994) A Microstructural Model for the Elastic Response of Articular Cartilage. Journal of Biomechanics, 27, 865-873. http://dx.doi.org/10.1016/0021-9290(94)90259-3

[27] Yutani, Y. and Yamano, Y. (1996) The Distribution of Differentiated Phenotypes of Chondrocytes in Osteoarthritic Cartilage. Osaka City Medical Journal, 42, 37-44.

[28] Aigner, T., Reichenberger, E., Bertling, W., Kirsh, T., Stob, H. and von der Mark, K. (1993) Type X Collagen Expression in Osteoarthritic and Rheumatoid Articular Cartilage. Virchows Archives B Cell Pathology, 63, 205-211.

[29] Stockwell, R.A. (1991) Cartilage Failure in Osteoarthritis: Relevance of Normal Structure and Function. A Review. Clinical Anatomy, 4, 161-191. http://dx.doi.org/10.1002/ca.980040303

[30] Chevalier, X. (1993) Fibronectin, Cartilage and Osteoarthritis. Seminars in Arthritis and Rheumatism, 22, 307-318. http://dx.doi.org/10.1016/S0049-0172(05)80010-1

[31] Wuster, N.B. and Lust, G. (1982) Synthesis of Fibronectin in Normal and Osteoarthritic Articular Cartilage. Biochemical and Biophysical Research Communications, 109, 1094-1101. http://dx.doi.org/10.1016/0006-291X(82)91889-7

[32] Paulsson, M. (1994) Non-Collagenous Matrix Proteins in Cartilage: Physiological Function and Diagnostic Usefulness in Joint Disease. Zeitschrift für Rheumatologie, 53, 1-96.

[33] Hardingham, T.E., Venn, G. and Bayliss, M.T. (1991) Chondrocyte Responses in Cartilage and in Experimental Osteoarthritis. British Journal of Rheumatology, 30, 32-37.

[34] Carney, S.L., Billingham, M.E.J., Caterson, B., Ratcliffe, A., Bayliss, M.T., Hardingham, T.E., et al. (1992) Changes 
in Proteoglycan Turnover in Experimental Canine Osteoarthritic Cartilage. Matrix, 12, 137-147. http://dx.doi.org/10.1016/S0934-8832(11)80055-7

[35] Dunham, J., Chambers, M.G., Jasani, M.K., Bitensky, L. and Chayen, J. (1990) Changes in the Orientation of Proteoglycans during the Early Development of Natural Murine Osteoarthritis. Journal of Orthopaedic Research, 8, 101104. http://dx.doi.org/10.1002/jor.1100080113

[36] Souza, R.B., Kumar, D., Calixto, N., Singh, J., Schooler, J., Subburaj, K., et al. (2014) Response of Knee Cartilage $\mathrm{T}_{1 \text { rho }}$ and $\mathrm{T}_{2}$ Relaxation Times to in Vivo Mechanical Loading in Individuals with and without Knee Osteoarthritis. Osteoarthritis and Cartilage. http://dx.doi.org/10.1016/j.joca.2014.04.017

[37] Mankin, H.J. and Brandt, K.D. (1989) Pathogenesis of Osteoarthritis. In: Kelley, W.N., Harris, E.D., Ruddy, S. and Sledge, C.B., Eds., Textbook of Rheumatology, 3rd Edition, WB Saunders Company, Philadelphia, 1469-1479.

[38] Chrisman, O.D., Ladenbauer-Bellis, I.M., Panjabi, M. and Goeltz, S. (1981) The Relationship of Mechanical Trauma and the Early Biochemical Reactions of Osteoarthritic Cartilage. Clinical Orthopaedics and Related Research, 161, 275-284.

[39] Kuettner, K.E. and Pauli, P.V. (1983) Vascularity of Cartilage. In: Hall, B.K., Ed., Cartilage, Vol. 1, Academic Press, New York, 281-312. http://dx.doi.org/10.1016/B978-0-12-319501-2.50016-9

[40] Borghetti, P., Salda, L.D., De Angleis, E., Maltarello, M.C., Petronini, P.G., Cabassi, E., et al. (1995) Adaptive Cellular Response to Osmotic Stress in Pig Articular Chondrocytes. Tissue and Cell, 27, 173-183. http://dx.doi.org/10.1016/S0040-8166(95)80020-4

[41] Brocklehurst, R., Bayliss, M.T., Maroudas, A., Coysh, H.L., Freeman, M.A.R., Revell, P.A., et al. (1984) The Composition of Normal and Osteoarthritic Articular Cartilage from Human Knee Joints. With Special Reference to Unicompartmental Replacement and Osteotomy of the Knee. Journal of Bone and Joint Surgery, 66, 95-106.

[42] Wei, F., Moore, D.C., Li, Y., Zhang, G., Wei, X., Lee, J.K. and Wei, L. (2012) Attenuation of Osteoarthritis via Blockade of the SDF-1/CXCR4 Signaling Pathway. Arthritis Research and Therapy, 14, R177. http://dx.doi.org/10.1186/ar3930

[43] Silacci, P., Dayer, J.M., Desgeorges, A., Peter, R., Manueddu, C. and Guerne, P.A. (1998) Interleukin (IL)-6 and Its Soluble Receptor Induce TIMP-1 Expression in Synoviocytes and Chondrocytes and Block IL-1-Induced Collagenolytic Activity. Journal of Biological Chemistry, 273, 13625-13629. http://dx.doi.org/10.1074/jbc.273.22.13625

[44] Roughly, P.J., Nguyen, Q. and Mort, J.S. (1991) Mechanisms of Proteoglycan Degradation in Human Articular Cartilage. Journal of Rheumatology, 18, 52-54.

[45] Sokoloff, L. (1987) Osteoarthritis as a Remodeling Process. Journal of Rheumatology, 14, 7-10.

[46] Benya, P.D. and Shaffer, J.D. (1982) Dedifferentiated Chondrocytes Reexpress the Differentiated Collagen Phenotype when Cultured in Agarose Gels. Cell, 30, 215-224. http://dx.doi.org/10.1016/0092-8674(82)90027-7

[47] Mobasheri, A., Kalamegam, G., Musumeci, G. and Batt, M.E. (2014) Chondrocyte and Mesenchymal Stem Cell-Based Therapies for Cartilage Repair in Osteoarthritis and Related Orthopaedic Conditions. Maturitas, 78, 188-198. http://dx.doi.org/10.1016/j.maturitas.2014.04.017

[48] Pettersen, I., Figenschau, Y., Olsen, E., Bakkelund, W., Smedsröd, B. and Sveinbjörnsson, B. (2002) Tumor Necrosis Factor-Related Apoptosis-Inducing Ligand Induces Apoptosis in Human Articular Chondrocytes in Vitro. Biochemistry and Biophysics Research Communications, 29, 671-676. http://dx.doi.org/10.1016/S0006-291X(02)00916-6

[49] Gelse, K., Aigner, T., Stöve, J. and Schneider, H. (2005) Gene Therapy Approaches for Cartilage Injury and Osteoarthritis. Current Medicinal Chemistry Anti-Inflammatory and Anti-Allergy Agents, 4, 265-279. http://dx.doi.org/10.2174/1568014054065203

[50] Wang, Q., Yang, Y.-Y., Niu, H.-J., Zhang, W.-J., Feng, Q.-J. and Chen, W.-F. (2013) An Ultrasound Study of Altered Hydration Behaviour of Proteoglycan-Degraded Articular Cartilage. BMC Musculoskeletal Disorders, 14, 1-18.

[51] Gao, Y., Liu, S., Huang, J., Guo, W., Chen, J., Zhang, L., et al. (2014) The ECM-Cell Interaction of Cartilage Extracellular Matrix on Chondrocytes. Biomedical Research International, 2014, Artical ID: 648459.

[52] Aigner, T., Sachse, A., Gebhard, P. and Roach, H. (2006) Osteoarthritis: Pathobiology-Targets and Ways for Therapeutic Intervention. Advanced Drug Delivery Reviews, 58, 128-149. http://dx.doi.org/10.1016/j.addr.2006.01.020

[53] Chang, C., Kuo, T., Wang, W., Wang, J.H., Hsu, Y.M., Huang, H.T., et al. (2011) Tissue Engineering-Based Cartilage Repair with Mesenchymal Stem Cells in a Porcine Model. Journal of Orthopaedic Research, 29, 1874-1880

[54] Wan, R., Hu, J., Zhou, Q., Wang, J., Liu, P. and Wei, Y. (2012) Application of Co-Expressed Genesto Articular Cartilage: New Hope for the Treatment of Osteoarthritis. Molecular Medicine Report, 6, 16-18. http://dx.doi.org/10.3892/mmr.2012.859

[55] Bhosale, A.M. and Richardson, J.B. (2008) Articular Cartilage: Structure, Injuries and Review of Management. British Medical Bulletin, 87, 77-95. http://dx.doi.org/10.1093/bmb/ldn025 
[56] Wei, F., Zhou, J., Wei, X., Zhang, J., Fleming, B.C., Terek, R., et al. (2012) Activation of Indian Hedgehog Promotes Chondrocyte Hypertrophy and Upregulation of MMP-13 in Human Osteoarthritic Cartilage. Osteoarthritis and Cartilage, 20, 755-763. http://dx.doi.org/10.1016/j.joca.2012.03.010

[57] Issa, R.I. and Griffin, T.M. (2012) Pathobiology of Obesity and Osteoarthritis: Integrating Biomechanics and Inflammation. Pathobiology of Aging and Age Related Diseases, 2, 17470.

[58] Minas, T. and Nehrer, S. (1997) Current Concepts in the Treatment of Articular Cartilage Defects. Orthopedics, 20, 525-538.

[59] Aigner, T., Söder, S., Gebhard, P.M., McAlinden, A. and Haag, J. (2007) Mechanisms of Disease: Role of Chondrocytes in the Pathogenesis of Osteoarthritis-Structure, Chaos and Senescence. Nature Clinical Practice Rheumatology, 3, 391-399. http://dx.doi.org/10.1038/ncprheum0534

[60] Brady, M.A., Waldman, S.D. and Ethier, C.R. (2014) The Application of Multiple Biophysical Cues to Engineer Functional Neocartilage for Treatment of Osteoarthritis (Part I: Cellular Response). Tissue Engineering Part B Review. 
Scientific Research Publishing (SCIRP) is one of the largest Open Access journal publishers. It is currently publishing more than 200 open access, online, peer-reviewed journals covering a wide range of academic disciplines. SCIRP serves the worldwide academic communities and contributes to the progress and application of science with its publication.

Other selected journals from SCIRP are listed as below. Submit your manuscript to us via either submit@scirp.org or Online Submission Portal.
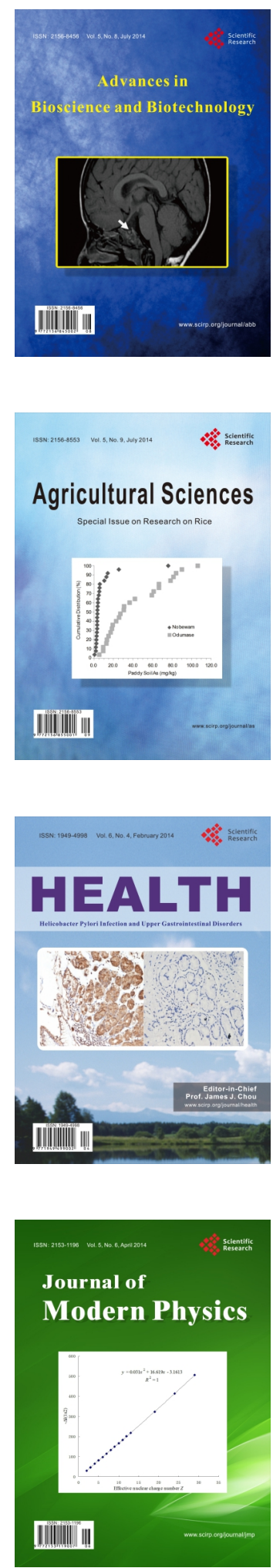
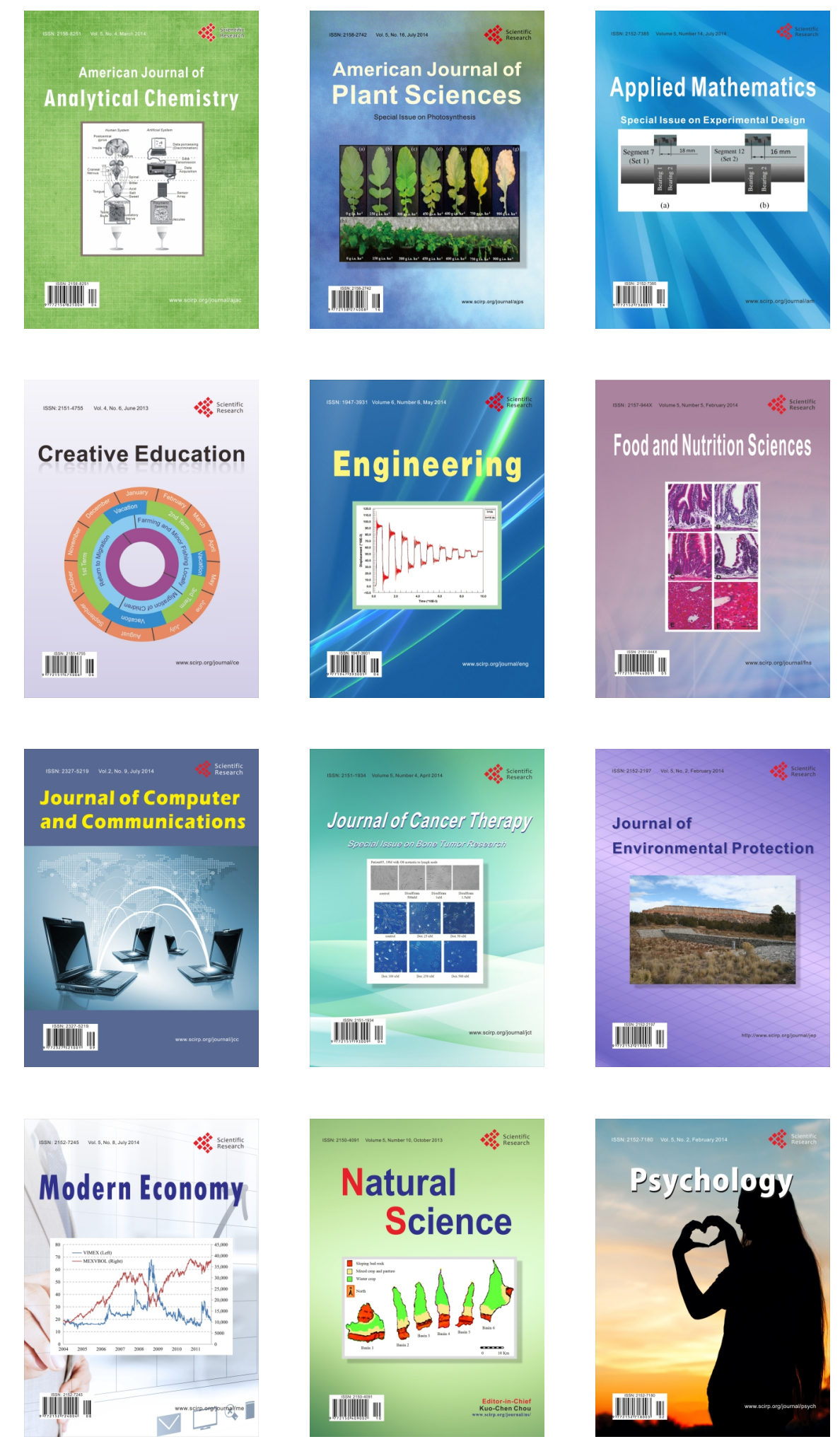